\title{
Functional and Pain Improvement in Tennis Elbow with Dry Needling as Alternative Treatment: Case Series
}

\author{
Deby Wahyuning Hadi, ${ }^{1}$ Henry Sugiharto ${ }^{2}$ and Amanda Tiksnadi ${ }^{3}$ \\ 1. Neurology Department, Faculty of Medicine, Airlangga University, Dr Soetomo General Hospital, Surabaya, Indonesia; \\ 2. Neurology Department, Faculty of Medicine, Sriwijaya University, Moh. Hoesin General Hospital, Sumatra, Indonesia; \\ 3. Neurology Department, Faculty of Medicine, University of Indonesia, Cipto Mangunkusumo General Hospital, Jakarta, Indonesia
}

DOI: https://doi.org/10.17925/USN.2021.17.1.60

ntroduction: Lateral epicondylitis, or tennis elbow, is pain in the lateral elbow as a result of repetitive forearm pronation and supination in elbow extension. Treatment varies from anti-inflammatory to surgical treatment. Dry needling has recently been developed as

a therapeutic modality in lateral epicondylitis; it is less invasive and can reduce the cost and time taken to carry out the procedure compared with other pain interventions or surgery. Case series: This case series reports three female patients, aged 38-42, with lateral elbow pain. Lateral epicondylitis diagnosis was based on medical history and palpatory findings, and was confirmed with provocative tests. Dry needling was performed on the extensor carpi radialis brevis (ECRB) and lateral epicondyle periosteum of all three patients. Two patients also had trigger points on the ECRB muscle, and therefore underwent needling on this location. Patients were evaluated using the functional and pain Patient-Rated Tennis Elbow Evaluation (fPRTEE and pPRTEE) scoring systems. Improvements to total pain resolution were obtained at the first evaluation (first week) until the last evaluation (fourth week post-treatment). Mean baseline fPRTEE score decreased from $72.0 \pm 7.1$ standard deviation (SD) to $34.0 \pm 5.4$ SD at the first evaluation. PPRTEE score also decreased from $33.3 \pm 1.2$ SD at baseline to $14.7 \pm 2.1 \mathrm{SD}$ at the first evaluation. Both scores were then 0 in the last evaluation. Conclusion: The improvement in patient-reported pain in these cases shows that dry needling could be considered as one of the treatment options for lateral epicondylitis. Further research is needed to support its clinical significance and efficacy.

\section{Keywords}

Tennis elbow, lateral epicondylitis,

dry needling, cases series

Disclosure: Deby Wahyuning Hadi, Henry

Sugiharto and Amanda Tiksnadi have no financial or non-financial relationships or activities

to declare in relation to this article.

Review process: Double-blind peer review.

Compliance with ethics: Informed consent was received from the patients involved in this case series.

Authorship: The named authors meet the International Committee of Medical Journal Editors (ICMJE) criteria for authorship of this manuscript, take responsibility for the integrity of the work as a whole, and have given final approval for the version to be published.

Access: This article is freely accessible at

touchNEUROLOGY.com (c) Touch Medical Media 2021

Received: 8 July 2020

Accepted: 27 April 2021

Published online: 2 August 2021

Citation: touchREVIEWS in Neurology. 2021;17(1):60-3

Correspondending author: Deby Wahyuning Hadi, Neurology Department, Faculty of Medicine, Airlangga University, Dr Soetomo General Hospital, 6-8 15th Mayjend Prof Dr. Moestopo, Surabaya, East Java, 16 Indonesia, 60286. E: deby.w.hadi@gmail.com
Lateral epicondylitis, or tennis elbow, is pain in the lateral elbow as a result of repetitive forearm pronation and supination in elbow extension. ${ }^{1}$ It is a frequent cause of elbow pain, and affects $1-3 \%$ of the adult population every year, resulting in decreased productivity and economic Iosses. ${ }^{2,3}$ Equally present in males and females, it mostly occurs between the age of 35 and 50 , and is more frequent in the dominant arm. ${ }^{2-4}$ The classical description of lateral epicondylitis relates to the sport of tennis, but only $5-10 \%$ of the patients who present with epicondylitis play this sport. In fact, tendinosis of the elbow is more common among non-sports players. ${ }^{3}$

Recently, it has become clear that lateral epicondylitis is a degenerative disorder that compromises the extensor tendons, originating from the lateral epicondyle, extending infrequently to the joint. ${ }^{3}$ Even though the terms 'epicondylitis' and 'tendinitis' are used to describe tennis elbow, histopathological studies, like those of Nirschl, characterize this condition rather as a form of tendinosis with a fibroblastic and vascular response, named angiofibroblastic degeneration of epicondylitis. ${ }^{3}$

The diagnosis of lateral epicondylitis can be made by observing the patient's history and clinical examination. ${ }^{1}$ Patients report pain at the lateral elbow that radiates down the forearm. Patients also often complain of weakened grip and difficulty in lifting objects. Physical examination typically reveals a tender point medial and distal to the lateral epicondyle, as well as pain with wrist flexion extremes and resisted elbow extension. ${ }^{4}$ Imaging in lateral epicondylitis is not always essential. Radiographs are mostly used only to rule out other causes of elbow pain and are commonly found to be normal in this pathology. Sonography can be a cost-effective modality to examine tendon pathology. While magnetic resonance imaging is preferable to sonography, its high cost needs to be considered. ${ }^{1}$

The clinical tests for lateral epicondylitis aim to reproduce the pain experienced by the patient. One test is Cozen's test, which is done with the elbow flexed at 90 degrees and with the forearm in pronation. The patient is asked to perform active extension of the wrist against resistance imposed by the examiner. Complaints of pain in the lateral epicondyle and at the origin of the extensor musculature of the wrist and fingers are indicative of tennis elbow. Another test, known as Mill's test, is performed with the patient's hand closed, the wrist in dorsiflexion and the 
Table 1: Clinical classification of lateral epicondylitis phases ${ }^{6}$

\begin{tabular}{|l|l|}
\hline Phase & Description of pain changes \\
\hline I & Mild pain after activity, usually subsides within 24 hours \\
\hline II & Mild pain more than 48 hours after activity, no pain during activity, can be relieved with warm-up exercises, and subsides within 72 hours \\
\hline III & Mild pain before and during activity, no significant negative impact on the activities, and can be partially relieved with warm-up exercises \\
\hline IV & Mild pain accompanies activities of daily living and has a negative impact on the performance of activities \\
\hline V & $\begin{array}{l}\text { Harmful pain unrelated to activities of daily living, can have a great negative impact on them but does not prevent them. Complete rest necessary to } \\
\text { control the pain }\end{array}$ \\
\hline VI & Persistent pain despite complete rest, and can prevent activities of daily living \\
\hline VII & Consistent pain at rest, aggravated after activities, and disturbed sleep \\
\hline
\end{tabular}

Table 2: Patient characteristics and analysis of Patient-Rated Tennis Elbow Evaluation scores

\begin{tabular}{|c|c|c|c|c|}
\hline & Patient 1 & Patient 2 & Patient 3 & Mean $\pm S D$ \\
\hline Age (years) & 42 & 38 & 40 & $40.0 \pm 1.6$ \\
\hline Sex & Female & Female & Female & \\
\hline Occupation & Housewife & Housewife & Housewife & \\
\hline Mill's test & + & + & + & \\
\hline Cozen's test & + & + & + & \\
\hline Maudley's test & - & - & - & \\
\hline Baseline fPRTEE & 62 & 78 & 76 & $72.0 \pm 7.1$ \\
\hline First week fPRTEE & 33 & 28 & 41 & $34.0 \pm 5.4$ \\
\hline Second week fPRTEE & 21 & 17 & 26 & $21.3 \pm 3.7$ \\
\hline Third week fPRTEE & 9 & 0 & 11 & $6.7 \pm 4.8$ \\
\hline Fourth week fPRTEE & 0 & 0 & 0 & \\
\hline Baseline pPRTEE & 32 & 35 & 33 & $33.3 \pm 1.2$ \\
\hline First week pPRTEE & 15 & 12 & 17 & $14.7 \pm 2.1$ \\
\hline Second week pPRTEE & 7 & 4 & 8 & $6.3 \pm 1.7$ \\
\hline Third week pPRTEE & 3 & 0 & 4 & $2.3 \pm 1.7$ \\
\hline Fourth week pPRTEE & 0 & 0 & 0 & \\
\hline
\end{tabular}

Cozen's test + = pain in the lateral epicondyle and at the origin of the extensor musculature of the wrist and fingers due to active extension of the wrist against resistance imposed by the examiner in position of elbow flexed at 90 degrees and with the forearm in pronation; fPRTEE = functional Patient-Rated Tennis Elbow Evaluation; Maudley's test $=$ no pain during resisted supination and resisted long finger extension; Mill's test $+=$ pain in the lateral epicondyle when the examiner forces the patient's wrist into flexion while the patient is asked to resist this movement with their hand closed; pPRTEE = pain Patient-Rated Tennis Elbow Evaluation; SD = standard deviation.

elbow extended. The examiner forces the wrist into flexion while the patient is asked to resist this movement. Pain in the lateral epicondyle confirms the positive results. ${ }^{4}$ Pain with resisted supination and resisted long finger extension (Maudsley's test) is suggestive of radial nerve entrapment at the radial tunnel and the ECRB, respectively. ${ }^{5}$ After confirmation of diagnosis, Nirschl and Ashman's classification system can be used to separate the disease into seven phases based on the level of pain and effects on function (Table 1). This system has since been used in research to show response to interventions. ${ }^{5-7}$

Corticosteroid injections, acupuncture, surgery and physiotherapy can be implemented as treatments for tennis elbow. Physiotherapy is a common treatment that is usually recommended for these patients. ${ }^{8}$

Nowadays, a new technique termed tendon needling, dry needling, needle tenotomy or tendon fenestration has been shown as an effective treatment for tendinitis, such as Achilles tendinitis, patellar tendinopathy and rotator cuff diseases. ${ }^{9,10}$ Repeated percutaneous needle fenestration of the affected tendon origin can possibly promote a healing response by disrupting the chronic degenerative process and encouraging localized bleeding and fibroblastic proliferation. This is thought to lead to ordered collagen formation and, ultimately, healing of the tendon.,.$^{911}$ Dry needling has been gradually applied to lateral epicondylitis, and there is evidence of its efficacy. ${ }^{11}$ This was detailed by Etminan et al., who reported a beneficial effect on formed tough bands, by creating contractions and twitch responses, and reducing spontaneous activity. ${ }^{8}$ The effects on blood circulation and increased oxygenation, and environmental neurophysiological effects, including secretion of opioids and betaendorphins, were used to control pain transfer. The mechanisms by which dry needling has been shown to work are central physiological effects, including segmental inhibition (gate control theory), opioid secretion, secretion of serotonin and noradrenaline neurotransmitters. ${ }^{8}$

The following cases describe three patients with tennis elbow, all of whom were successfully treated with dry needling.

\section{Case reports}

This case series includes three female patients, aged 38-42, with first time lateral elbow pain. All patients were housewives and performed daily housework themselves. Patients had never had any treatment or medication. They presented to our clinic on day 3-5 of symptom onset and were all classified as phase VI based on the clinical Nirschl classification. 
Figure 1: Dry needling procedure in patients

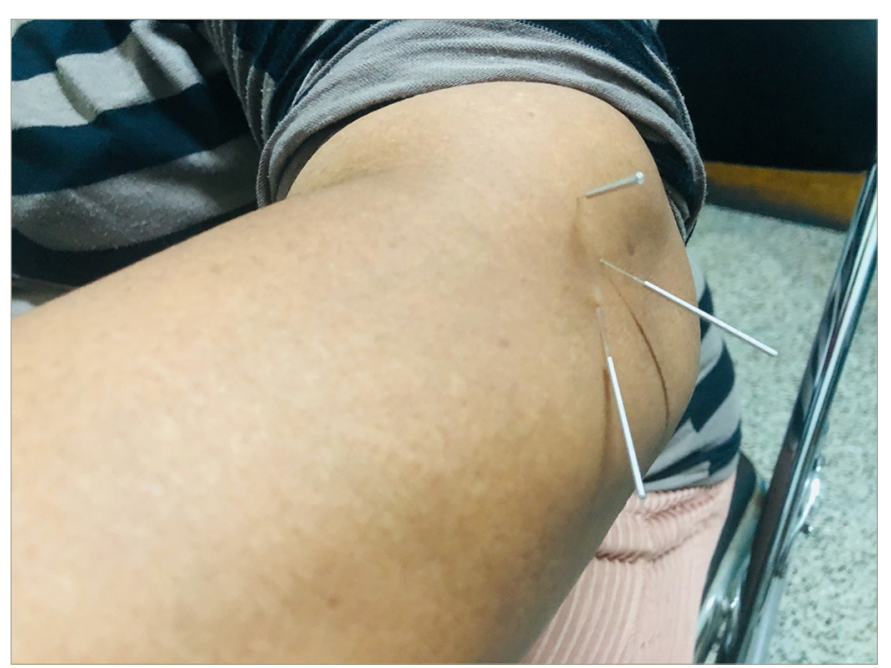

Lateral epicondylitis diagnosis was based on medical history (pain on lateral elbow and history of chronic stress to the forearm muscles with repetitive activities, i.e. gripping, wrist extension, radial deviation, forearm supination) and palpatory findings; diagnoses were confirmed with Mill's test and Cozen's test. Maudsley's test was also performed to rule out radial nerve entrapment. Pre- and post-dry needling evaluation was conducted using the pain and functional Patient-Rated Tennis Elbow Evaluation (PRTEE) scores.,212 Patients were followed-up weekly, until 4 weeks post-treatment. Patient characteristics are listed in Table 2.

The PRTEE scoring system has two subscales, functional (fPRTEE) and pain (PPRTEE). The pain subscale consists of 5 items, while the functional subscale has 10 items; each item has a maximum of 10 points. Therefore, 50 is the maximum PPRTEE score and 100 is the maximum fPRTEE score. During the pPRTEE assessment, patients were asked to rate their pain during specific movements; while in fPRTEE, they had to rate the amount of difficulty involved in specific and usual activities.

\section{Dry needling procedure}

Dry needling was performed once during the initial visit, on an outpatient basis, by a neurologist. All procedures were conducted with the patient sitting in a chair with elbows at 90 degrees of flexion and the forearm in the neutral position. The procedure used 3-5 sterile, stainless steel, 40 $\mathrm{mm}$ long needles with a diameter of $0.25 \mathrm{~mm}$. Needles were inserted on the ECRB tendon and lateral epicondyle periosteum (tendon needling and periosteal pecking technique) by inspection and palpation, penetrated in and out 20-30 times without pulling them out of the skin, and then left in situ for 5 minutes (Figure 1). Two patients also had trigger points on the ECRB muscle, so needling was also carried out there. After the dry needling, we placed an ice pack around the area to reduce the soreness that may be associated with dry needling.

Improvements to total pain resolution were obtained at the first evaluation ( 1 week post-treatment) until the last evaluation (4 weeks post-treatment). No soreness post-needling was reported in any of the patients. Mean baseline fPRTEE score decreased from $72.0 \pm 7.1$ standard deviation (SD) at baseline to $34.0 \pm 5.4$ SD in the first evaluation (Figure 2 and Table 2). pPRTEE score also decreased from $33.3 \pm 1.2 \mathrm{SD}$ at baseline to $14.7 \pm 2.1 \mathrm{SD}$ at the first evaluation. Both scores were 0 at the last evaluation (Figure 3 and Table 2). Individual fPRTEE and PPRTEE scores for each patient are reported in Table 2.

\section{Figure 2: Functional Patient-Rated Tennis Elbow Evaluation}

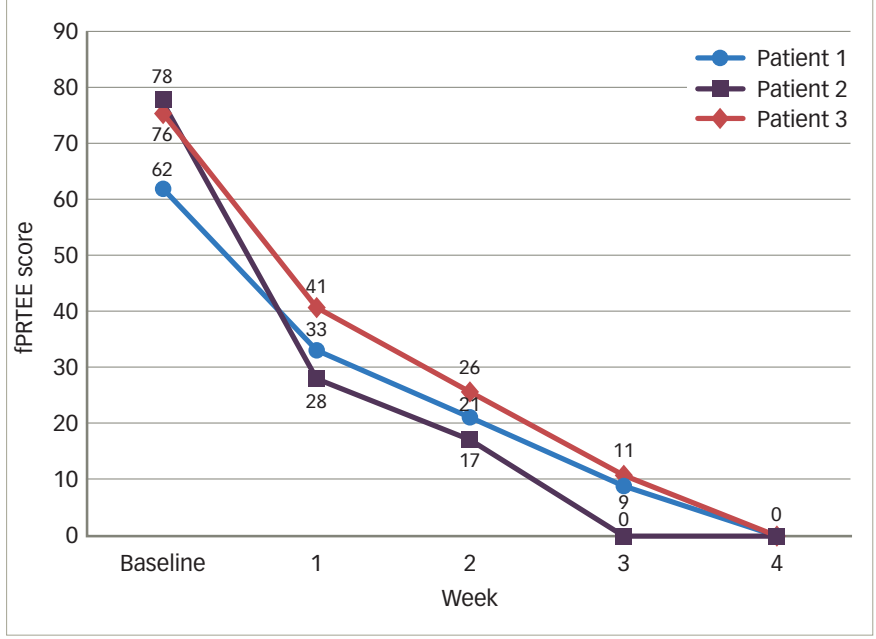

fPRTEE score $=$ functional Patient-Related Tennis Elbow Evaluation score.

Figure 3: Pain Patient-Rated Tennis Elbow Evaluation

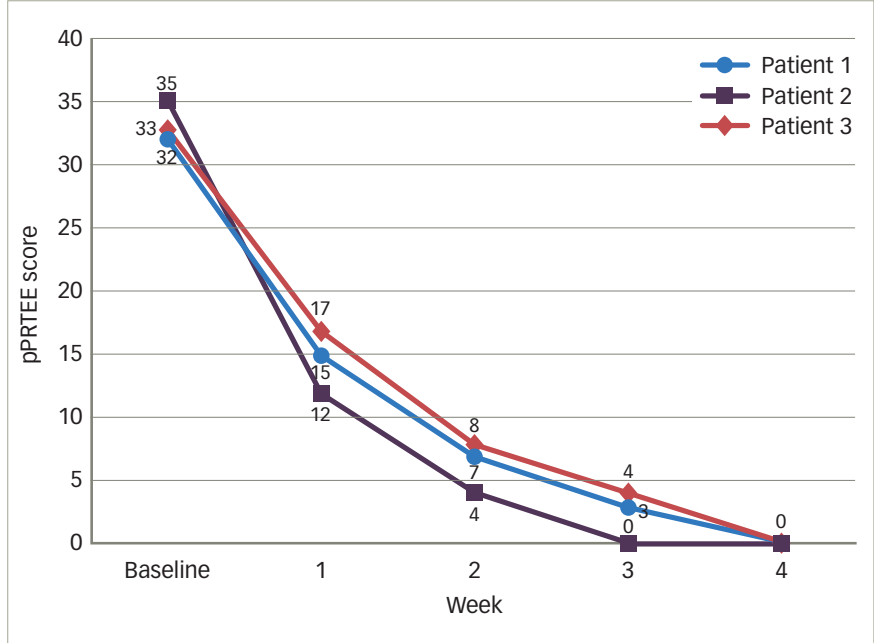

PPRTEE SCore = pain Patient-Related Tennis Elbow Evaluation score.

\section{Discussion}

The mean age of patients in this case series was $40.0 \pm 1.6$ SD, which is consistent with the epidemiological data from several previous studies. ${ }^{45,13}$ Most cases of lateral epicondylitis can be clinically confirmed by a detailed history and physical examination. Radiographic diagnosis reveals normal findings in most cases, but can be useful to rule out other abnormalities, such as arthrosis, osteochondritis dissecans or articular free bodies. However, there are some possible findings related to lateral epicondylitis, such as calcification of the ECRB origin. ${ }^{17}$ In this study, we did not perform $\mathrm{X}$-ray due to time constraints. X-ray was planned if the patients' pain did not improve, but all patients showed improvement during their follow-up.

Sonography is an efficient and non-invasive diagnostic tool for lateral epicondylitis. Possible findings for identifying degenerative changes of the tendons attached to the region of the lateral epicondyle include bone irregularities; calcific deposit; and thickening, thinning and tears of affected tendons or capsules. Ultrasound can also be a useful tool for image-guided intervention. ${ }^{1.6}$ Ultrasound was not performed on the patients presented here due to lack of resource. If ultrasound had been required, we would refer the patients to another facility. 
Of our three patients, patient 2 had the highest baseline PPRTEE and fPRTEE scores, but she also experienced the fastest complete resolution. This might be related to the onset of symptoms; she presented on day 3 of onset, while the two other patients presented on day 5. A study by Smidt et al. confirmed that duration of complaints is one of the most consistent prognostic factors for the outcome of lateral epicondylitis, associated with worse outcomes at 12 months. ${ }^{14}$

Needling procedures in tendinopathy, specifically lateral epicondylitis, can vary in the number of needles, the manipulation technique and the duration of procedure. One might use the in-and-out technique, like we did in our study, and others may use a rotation technique, as performed by Uygur et al. ${ }^{2}$ In a case report, Shariat et al. only kept the needles in place for 20 minutes, without performing a manipulation technique. ${ }^{15}$ However, this technique yielded positive results in both pain and disability.

The pathophysiology of lateral epicondylitis is still being debated. New studies show that the main pathophysiological features of tendinopathy are neovascularity and irregular collagen fibres. ${ }^{9,1,15}$ However, the cause of degenerative changes and pain is unclear. Mechanical, neural and vascular problems, and failure of healing are presumed to contribute to the pathophysiology of this condition. ${ }^{9,16,17}$ In this case, needling was performed on the ECRB tendon and lateral epicondyle periosteum. In addition, in two cases, a trigger point was also found on the ECRB muscle, hence needling was also carried out on the trigger point.

Although the exact mechanism of dry needling is not yet clear, this technique essentially decreases peripheral and central sensitization, ${ }^{18-21}$ which certainly influences tendon healing due to increased blood flow through local vasodilation and collagen proliferation. 10,18,19 $^{1}$ One review stated that periosteal stimulation can cause microtrauma, trigger local inflammation, improve fibroblastic repair processes, increase concentration and reorganization of collagen tissue, and mediate the proliferative and remodelling phases. ${ }^{22}$

Current research provides initial support for the use of dry needling as a stand-alone procedure for tendinopathy. It seems that it is a safe and effective treatment method, although there is vast diversity regarding the technique of the needling, ultrasound guidance and additional treatments used. The long-term benefits of dry needling as a stand-alone treatment in lateral epicondylitis have been demonstrated in a study by McShane et al., which showed good or excellent outcomes in $92.3 \%$ of patients treated with percutaneous tenotomy as a sole treatment for refractory lateral epicondylitis at an average followup of 22 months. ${ }^{23}$ In contrast, Stenhouse et al. did not demonstrate significant differences in clinical outcomes between two treatment groups (dry needling group and autologous conditioned plasma group) at any follow-up interval; although, there was a trend towards greater clinical improvement in the autologous conditioned plasma group in the short term $(27.1 \%$ improvement in pain score at 2 months compared with $12.3 \%$ in the dry needling group). ${ }^{13}$

In the present case series, dry needling was performed only once at the patients' initial visit; however, we had actually prepared for a second procedure in the fourth week. The procedure became unnecessary, as pain and functional scales showed complete resolution at week 4. However, long-term follow-up might be necessary to gather long-term effect data. In contrast to our findings, one study that performed dry needling twice, with an interval of 1 month between procedures, noted a temporary clinical deterioration following the second dry needling procedure (compared with 1-month after the initial procedure). ${ }^{13}$ The reason for this is unclear; however, it is possible that by repeating the dry needling, it may interrupt the original healing cascade that was initiated previously. ${ }^{13}$

\section{Conclusion}

The three patients in our case series experienced the benefits of dry needling in terms of decreased pain and improved function after one procedure. Dry needling is minimally invasive, inexpensive, carries a low risk, and is quick and easy to administer. Given its promising long-term therapeutic effect in lateral epicondylitis, like those demonstrated in Suzuki's and Stenhouse's studies, ${ }^{11,13}$ a large randomized controlled trial is needed to validate these encouraging results. $\square$
1. Erdem Y, Neyisci C. Lateral and medial epicondylitis: definition, diagnosis, screening and treatment algorithms. In: Korhan o. Work-related and musculoskeletal disorders. London, UK: Intechopen, 2019;59

2. Uygur E, Aktas B, Ozkut A, et al. Dry needling in lateral epicondylitis: a prospective control study. Int Orthop. 2017;41:2321-5.

3. Nirschl RP, Pettrone FA. Tennis elbow. The surgical treatment of lateral epicondylitis. J Bone Joint Surg Am. 1979;61:832-9. Cohen M, da Rocha Motta Filho G. Lateral epicondylitis of the elbow. Rev Bras Ortop. 2012;47:414-20.

Faro F, Wolf JM. Lateral epicondylitis: review and current concepts. J Hand Surg Am. 2007;32:1271-9.

Nirschl RP, Ashman ES. Elbow tendinopathy: tennis elbow. Clin Sports Med. 2003;22:813-36.

Sports Med. $2003,22: 813-36$.
Ma K-L, Wang H-Q. Management of lateral of epicondylitis: a narrative literature review. Pain Res Manag. 2020;2020:6965381.

8. Etminan Z, Razeghi M, Ghafarinejad F. The effect of dry needling of trigger points in forearm's extensor muscles on the grip force, pain and function of athletes with chronic tennis elbow. Journal of Rehabilitation Sciences and Research. 2019;6;27-33.

9. Krey D, Borchers J, McCamey K. Tendon needling for treatment of tendinopathy: a systematic review. Phys Sportsmed. 2015;43:80-6.
10. Tsikopoulos K. Tsikopoulos I, Simeonidis E, et al. The clinical impact of platelet-rich plasma on tendinopathy compared to placebo or dry needling injections: A meta-analysis. Phys The Sport. 2016;17:87-94.

11. Suzuki T, Iwamoto T, Matsumura $N$, et al. Percutaneous tendon needling without ultrasonography for lateral epicondylitis. Keio J Med. 2019;69;37-42.

12. Rompe JD, Overend TJ, Macdermid JC. Validation of the Patientrated Tennis Elbow Evaluation questionnaire. I Hand Ther. 2007:3-11.

13. Stenhouse G, Sookur P, Watson M. Do blood growth factors offer additional benefit in refractory lateral epicondylitis? a prospective, randomized pilot trial of dry needling as a stand-alone procedure versus dry needling and autologous conditioned plasma. Skeletal Radiol. 2013;42:1515-20.

14. Smidt $\mathrm{N}$, Lewis $\mathrm{M}$, van der Windt $\mathrm{DA}$, et al. Lateral epicondylitis in general practice: course and prognostic indicators of in general practice: course and prognosic in
outcome. J Rheumatol. 2006;33:2053-9.

15. Shariat A, Noormohammadpour $P$, Memari AH, et al. Acute effects of one session dry needling on a chronic golfer's elbow disability. J Exerc Rehabil. 2018;14:138-42.

16. Mishra AK, Skrepnik NV, Edwards SG, et al. Efficacy of platelet-rich plasma for chronic tennis elbow: a double-blind, prospective, multicenter, randomized controlled trial of 230 patients. Am I Sports Med. 2014:42:463-71.

17. Rabago $D$, Best TM, Zgierska AE, et al. A systematic review of four injection therapies for lateral epicondylosis: prolotherapy, polidocanol, whole blood and platelet-ric plasma. Br J Sports Med. 2009;43:471-81.

18. Cagnie B, Dewitte V, Barbe T, et al. Physiologic effects of dry needling. Curr Pain Headache Rep. 2013;17:348

19. Dunning J, Butts R, Mourad F, et al. Dry needling: a literature review with implications for clinical practice guidelines. Phys Ther Rev. 2014;19:252-65.

20. Dommerholt J. Dry needling - peripheral and central considerations. J Man Manip Ther. 2011;19:223-7.

21. Ziaeifar M, Arab AM, Karimi N, Nourbakhsh MR. The effect of dry needling on pain, pressure pain threshold and disability in patients with a myofascial trigger point in the upper trapezius muscle. J Bodyw Mov Ther. 2014;18:298-305.

22. Dommerholt J, Chou LW, Finnegan M, Hooks T. A critical overview of the current myofascial pain literature. J Bodyw Mov Ther. 2019;23:295-305.

23. MCShane JM, Shah VN, Nazarian LN. Sonographically guided percutaneous needle tenotomy for treatment of common extensor tendinosis in the elbow: is a corticosteroid necessary? J Ultrasound Med. 2008;27:1137-44. 